\title{
Finger numeral representations: more than just another symbolic code
}

\author{
Samuel Di Luca and Mauro Pesenti* \\ Centre de Neuroscience Système et Cognition, Institut de Recherche en Sciences Psychologiques, Université catholique de Louvain, Louvain-la-Neuve, Belgium \\ *Correspondence: mauro.pesenti@uclouvain.be
}

Representing numerosities with finger configurations offers children the opportunity to learn and internalize fundamental properties of natural numbers through sensorymotor interactions with the world. Recent findings show that even educated adults use their fingers as a visuo-motor support to process, represent, and communicate numerosities. Indeed, using fingers to represent numerosities prototypically has been shown to give the corresponding finger configurations a special status in long-term memory: these configurations are recognized and processed faster than other finger configurations, providing a direct access to number magnitude, what other finger configurations do less efficiently. This occurs for configurations stemming from finger counting (i.e., the way the fingers are raised to count for oneself; for example, thumb, index, and middle fingers for numerosity \{3\}) and from finger montring (i.e., the way fingers are raised to show numerosities to someone else; for example, index, middle, and ring fingers for numerosity $\{3\})$. However, finger numeral representation is not just another way of representing numerical magnitudes mentally. We argue that it also contributes to acquiring, building, and then accessing number semantics, and that, compared to other numerical representations, it provides an extra value by rooting number meaning in a culturally shared yet non-arbitrary and self-experienced sensory-motor representation.

\section{ACQUIRING NUMERICAL KNOWLEDGE AND MATHEMATICAL CONCEPTS THANKS TO FINGERS}

Children from many human cultures use finger-counting strategies to enumerate sets of objects and use their fingers when solving mathematical tasks. They "visually" represent numerosities by raising the same number of fingers as the number of items counted and, by doing this, they get a finger configuration preserving the cardinality of the set. Using finger counting is the first or second most frequent strategy observed in preschoolers during counting and arithmetical tasks (Fuson, 1982), importantly even when no explicit instructions to use their fingers have been given (Siegler and Shrager, 1984). Their use during basic arithmetical learning has been extensively studied (e.g., Fuson, 1988), and internal traces of these external strategies may still affect calculation in children even when finger counting is no longer overtly used. For example, children's typical split-5 errors in addition and subtraction (e.g., $12-5=2$ ) may stem from an interiorized finger-counting strategy using a sub-base 5 represented with a full-opened hand (Domahs et al., 2008). Using the same finger configuration repeatedly to represent a given numerosity for oneself or to show it to others gives this configuration a special iconographic status and, as shown below, also a symbolic one. Likewise, the use of a stable, culturally determined - and probably partly constrained at the motor level - sequence of finger movements while counting allows children to remember the sequence of counted elements by establishing a one-to-one correspondence between the raised fingers and the objects, and to better understand and develop numerical concepts such as cardinality and ordinality, or the first element and unique immediate successor-predecessor principles. For these reasons, finger counting has been considered as a mediator between an inner rough number sense and a developed, symbolically represented, number concept (Fayol and Seron, 2005; Andres et al., 2008). It explains why tactile discrimination is strongly related to arithmetical competencies. As a matter of fact, the score obtained in finger discrimination tasks is the best predictor of arithmetical performance in 5- to 8-year old children (Fayol et al., 1998; Marinthe et al., 2001; Noël, 2005), and training in finger differentiation to increase finger gnosis can improves untrained mathematical skills (Gracia-Bafalluy and Noël, 2008).
This influence of fingers on the acquisition of numbers and numerical concepts is also indicated by several historical and linguistic facts. Indeed, "handling" numerosities not only improved human mathematical competencies (Butterworth, 1999), it probably gave rise to our positional base10 numerical system rather than others (e.g., a base-12 one), which possess some arithmetical advantages (e.g., more divisors) and are thus more suitable for geometric calculus and algebra. This occurred not in a few limited cultural groups, but in many different cultures across human history. There are numerous archeological traces (e.g., artifacts such as reliefs and mosaics) of finger-counting strategies in ancient cultures (Boyer, 1968; Ifrah, 1981), and numerous references to finger counting or finger calculation in Greek and Roman manuscripts (Williams and Williams, 1995). Around two thirds of several hundred Native American tribes used base- 5 or base- 10 systems derived from finger counting (Eels, 1913; cited in Boyer, 1968), and several studies have described in detail how indigenous Papua New Guineans use their fingers and body parts while counting (Lancy, 1978; Saxe, 1982). An additional piece of evidence concerns the origin of number names themselves. In various languages, number names stem from an ancient embodied vocabulary referring to fingers (e.g., in English, digit means at the same time number and finger; five comes from a common root of finger and fist; Menninger, 1969), supporting the idea that counting originates from the use of fingers rather than from arbitrary quantitative words.

\section{ACCESSING NUMBER SEMANTICS THROUGH FINGER NUMERAL REPRESENTATIONS DURING ADULTHOOD}

Besides these developmental and cultural pieces of evidence, recent findings in adults show that finger counting shapes number processing and calculation throughout life, 
and that finger numeral representations do not disappear when symbolic numerical representations develop. On the contrary, their critical impact is still observed in educated adults.

Firstly, finger-counting strategies influence the way numerical information is projected onto physical space and induces compatibility effects, at least at the level of motor outputs. For example, personal finger-counting habits were found to actively interact with Arabic digit processing during a number-to-finger mapping task. When asked to identify Arabic digits by pressing a key with 1 of their 10 fingers, participants produced faster responses when the mapping between the Arabic digits and the fingers matched their own finger-counting habits than with other mappings (Di Luca et al., 2006). This is also evidenced in parity judgments by a specific increase in motorevoked potentials for the right hand only when small numerosities are processed by adults who show a prototypical fingercounting sequence starting with the right hand (Sato et al., 2007). Personal fingercounting habits could even mediate the well-known association between space and numbers (i.e., small numbers being associated with the left space, and larger numbers with the right; Dehaene et al., 1993), as they seem to modulate the strength of this association (Fischer, 2008).

Next, finger numeral representations exert their influence even when no motor outputs are required. For example, just like children (Noël, 2005), adults name finger configurations faster when they conform to their own finger-counting habits than when they do not (Di Luca and Pesenti, 2008). This facilitation in the naming of canonical configurations is not a mere perceptual effect but truly reflects semantic access. Indeed, numeral finger configurations used as unconsciously presented primes influence comparative judgments of Arabic numeral targets: the participants respond faster to and make fewer errors with numerical than with non-numerical primes, and when the primes and targets are congruent (i.e., lead to the same response), but this priming effect generalizes to new, never-consciously seen, numerosities for canonical configurations only, not to non-canonical ones. Furthermore, mere visuo-perceptual differences are not the source of the better identification of and semantic access to canonical configurations. When participants have to decide whether a canonical configuration is present among a set of distractors expressing the same numerosity in a non-canonical way, the time to detect the presence of the target grows linearly with the number of distractors showing that canonical targets enjoy no perceptual saliency (i.e., no pop-out effect; Di Luca and Pesenti, 2010). Most interestingly, a recent study shows that canonical configurations are processed in the same way as other symbolic notations (Di Luca et al., 2010). When participants named Arabic and verbal numerals primed by canonical and non-canonical finger numeral configurations, canonical configurations primed target numbers to which they were close, whether they were smaller or larger than the target, with the extent of activation being inversely proportional to the distance between the prime and the target. This results in a $V$-shaped pattern of priming, supporting the idea that canonical configurations, although not supported by a written system, activate representations with the same properties as those activated by verbal or Arabic numerals (i.e., a placecoding representation; Roggeman et al., 2007).

Finally, finger numeral representations also have an impact on arithmetic. In a recent study (Badets et al., 2010), participants provided a verbal response to simple additions, which triggered the presentation of a correct or incorrect result displayed either as canonical configurations of fingers or as a series of rods. They answered more quickly with finger configurations than with rods, but only when the finger configurations showed the correct response. This supports the idea that, even in adults, simple arithmetic operations are still unconsciously underpinned by finger numeral representations.

\section{EXTRA VALUE CODE?}

Given these findings in children and adults, finger numeral representations (whether they come from finger counting, finger montring, or other personal ways of using fingers to represent numerosities) certainly qualify as another type of numerical representation worthy of being considered by current cognitive numberprocessing architectures - perhaps as a fourth type of representation if they were to be integrated into Dehaene's (1992)
Triple Code framework. But is that all? Are finger numeral representations nothing but another way of representing numbers, mainly small ones, mentally? What, after all, makes them so special for numerical cognition?

We believe and argue that finger numeral representations are more than just another way of mentally representing numerosities. Firstly, they possess almost all the properties presented separately by the other representations (i.e., visual, verbal, and analog). Although they are optimal only for small numerosities and they are not linked to a written notation, they possess simultaneously iconic (i.e., features shared with the referent), symbolic (i.e., conventional meaning shared with other individuals), computational (i.e., used to support calculation procedures), and communicative (i.e., used to communicate numerosities through gestures with other individuals whatever their language) properties. Secondly, and most importantly, all these properties rely on perceptual and sensory-motor processes that provide a non-arbitrary link between the symbols (here, finger configurations) and reality (here, numerosity), and that can be spontaneously self-experienced by every human child and adult. In contrast, other representations only possess some of these properties and they cannot be inferred and acquired without external influence. Visual and verbal representations serve a communicative purpose because they are shared among individuals, but they possess no numerical meaning and very little can be inferred from them by the cognitive system as they stand for symbolic notations (respectively, verbal and Arabic numerals) composed of totally arbitrary symbols. For example, "6" and "six" can unambiguously be communicated and understood, but no numerical meaning can be inferred from either their physical traits, or their mental representation. An analog number line can easily represent continuous and large numerical quantities and their ratio, but it cannot easily serve the purpose of accurate communication. Moreover, except in very few people who explicitly develop a spatio-linear representation of numbers (Galton, 1880; Seron et al., 1992), there is no evidence of a spontaneously self-practiced linear medium underlying and guiding early numerical learning. Rather, early 
numerical competencies may simply rely on some perceptual object-tracking system (Simon, 1997; Uller et al., 1999; Mix et al., 2002), the association between numbers and space leading to a linear representation being constructed by exposure to cultural conventions (Dehaene, 1997; Simon, 1999), such as reading-writing direction. By contrast, the very act of using fingers to represent numerosities seems quite spontaneous - what is culturally determined is the sequence in which fingers are raised - and can guide early numerical learning. In other words, a number line is most probably the best conceptual representation induced by the cultural environment, whereas finger numeral representations are the best empirical representation, which can be deduced from personal sensorymotor experience.

\section{CONCLUSION}

Recent findings show that finger numeral representations possess many characteristics of the other numerical representations postulated in classical cognitive number architectures. Among others, they, like other symbolic notations, are shared by individuals of the same cultural group, they can be used to communicate numerosities and to calculate, they possess iconic properties preserving cardinality, and place-coding properties. Most importantly, they have specific sensorymotor properties preserving numerical properties and allowing mathematical principles to be inferred and experienced. Thus, they are not just a way of mentally representing (in the sense of "standing for") numerosities as other representations do; they represent and, at the same time, can help to build or, at least, improve the concept of number. We do not intend to claim that finger numeral representations replace all other representations, or that without finger-counting activities, human beings could not develop an accurate concept of number. But fingercounting/montring activities, especially if practiced at an early age, can contribute to a fast and deep understanding of number concepts, which has an impact during the entire cycle of life by providing the sensory-motor roots onto which the number concept grows.

\section{ACKNOWLEDGMENTS}

Samuel Di Luca is a post-doctoral researcher and Mauro Pesenti a research associate at the National Fund for Scientific Research (Belgium).

\section{REFERENCES}

Andres, M., Di Luca, S., and Pesenti, M. (2008). Finger counting: the missing tool? Behav. Brain Sci. 31, 642-643.

Badets, A., Pesenti, M., and Olivier, E. (2010). Responseeffect compatibility of finger-numeral configurations in arithmetical context. Q. J. Exp. Psychol. 63, 16-22.

Boyer, C. B. (1968). A History of Mathematics. New York, NY: John Wiley and Sons.

Butterworth, B. (1999). The Mathematical Brain. London: Macmillan.

Dehaene, S. (1992). Varieties of numerical abilities. Cognition 44, 1-42.

Dehaene, S. (1997). The Number Sense. Oxford: Oxford University Press.

Dehaene, S., Bossini, S., and Giraux, S. (1993). The mental representation of parity and number magnitude. $J$. Exp. Psychol. Gen. 122, 371-396.

Di Luca, S., Granà, A., Semenza, C., Seron, X., and Pesenti, M. (2006). Finger-digit compatibility in Arabic numerical processing. Q. J. Exp. Psychol. 59, 1648-1663.

Di Luca, S., Lefèvre, N., and Pesenti, M. (2010). Place and summation coding respectively for canonical and non-canonical finger numeral representations. Cognition 117, 95-100.

Di Luca, S., and Pesenti, M. (2008). Masked priming effect with canonical finger numeral configurations. Exp. Brain Res. 185, 27-39.

Di Luca, S., and Pesenti, M. (2010). Absence of low-level visual difference between canonical and non-canonical finger-numeral configurations. Exp. Psychol. 57, 202-207.

Domahs, F., Krinzinger, H., and Willmes, K. (2008). Mind the gap between both hands: evidence for internal finger-based number representations in children's mental calculation. Cortex 44, 359-367.

Eels, W. C. (1913). Number systems of North American Indians. Am. Math. Mon. 20, 293.

Fayol, M., Barrouillet, P., and Marinthe, C. (1998) Predicting arithmetical achievement from neuropsychological performance: a longitudinal study. Cognition 68, 63-70.

Fayol, M., and Seron, X. (2005). “About numerical representations: insights from neuropsychological, experimental and developmental studies," in Handbook of Mathematical Cognition, ed. J. I. D. Campbell (New York: Psychology Press), 3-22.

Fischer, M. H. (2008). Finger counting habits modulate spatial-numerical associations. Cortex 44, 386-392.

Fuson, K. C. (1982). "An analysis of the counting on-solution procedure in addition," in Addition and Subtraction: A Cognitive Perspective, eds T. P. Carpenter, J. M. Moser, and T. A. Romberg (Hillsdale: LEA), 67-81.

Fuson, K C. (1988). Children's Counting and the Concepts of Number. New York: Springer.

Galton, F. (1880). Visualised numerals. Nature 21, 252-256.
Gracia-Bafalluy, M., and Noël, M. P. (2008). Does finger training increase young children's numerical performance? Cortex 44, 544-560.

Ifrah, G. (1981). Histoire universelle des chiffres. Paris: Robert Laffont.

Lancy, D. F. (1978). Cognitive testing in the indigenous mathematics project. Papua New Guinean J. Edu. 14, 114-142.

Marinthe, C., Fayol, M., and Barrouillet, P. (2001). "Gnosies digitales et développement des performances arithmétiques," in Troubles du Calcul et Dyscalculies chez l'Enfant, eds A. Van Hout, C. Meljac, and J. P. Fischer (Paris: Masson), 239-254.

Menninger, K. (1969). Number Words and Number Symbols. Cambridge, MA: MIT Press.

Mix, K. S., Huttenlocher, J., and Levine, S. C. (2002). Multiple cues for quantification in infancy: is number one of them? Psychol. Bull. 128, 278-294.

Noël, M.P. (2005). Finger gnosia: a predictor of numerical abilities in children? Child Neuropsychol. 11,413-430.

Roggeman, C., Verguts, T., and Fias, W. (2007). Priming reveals differential coding of symbolic and non-symbolic quantities. Cognition 105, 380-394.

Sato, M., Cattaneo, L., Rizzolatti, G., and Gallese, V. (2007). Numbers within our hands: modulation of cortico-spinal excitability of hand muscles during numerical judgement. J. Cogn. Neurosci. 19, 684-693.

Saxe, G. B. (1982). "Culture and the development of numerical cognition: studies among the Oksapmin of Papua New Guinea," in Children's Logical and Mathematical Cognition, ed.C. J. Brainerd (New York, NY: Springer-Verlag), 157-176.

Seron, X., Pesenti, M., Noël, M. P., Deloche, G., and Cornet, J. A. (1992). Images of numbers, or "When 98 is upper left and 6 sky blue". Cognition 44, 159-196.

Siegler, R. S., and Shrager, J. (1984). "Strategy choices in addition and subtraction: how do children know what to do?" in Origins of Cognitive Skills, ed. C. Sophian (Hillsdale: LEA), 229-293.

Simon, T. J. (1997). Reconceptualizing the origins of number knowledge: a "nonnumerical" account. Cogn. Dev. 12, 349-372.

Simon, T. J. (1999). The foundation of numerical thinking in a brain without numbers. Trends Cogn. Sci. 3, 363-365.

Uller, C., Huntley-Fenner, G., Carey, S., and Klatt, L. (1999). What representations might underlie infant numerical knowledge? Cogn. Dev. 14, 1-36.

Williams, B. P., and Williams, R.S. (1995). Finger numbers in the Greco-Roman world and the early Middle Ages. Isis 86, 587-608.

Received: 28 June 2011; accepted: 28 September 2011; published online: 04 November 2011.

Citation: Di Luca S and Pesenti M (2011) Finger numeral representations: more than just another symbolic code. Front. Psychology 2:272. doi: 10.3389/fpsyg.2011.00272 This article was submitted to Frontiers in Cognition, a specialty of Frontiers in Psychology.

Copyright (C) 2011 Di Luca and Pesenti. This is an openaccess article subject to a non-exclusive license between the authors and Frontiers Media SA, which permits use, distribution and reproduction in other forums, provided the original authors and source are credited and other Frontiers conditions are complied with. 